\title{
Gestión de riesgos en las gerencias de seguridad industrial de la industria petrolera venezolana
}

\author{
Risk management in the industrial safety management of the \\ venezuelan oil industry
}

\author{
Andy Leal \\ andyjoseleal@gmail.com \\ ORCID 0000-0002-2278-7284
}

Empresa Mixta Petro-Boscán, Venezuela

Artículo recibido mayo 2019 | Arbitrado en julio 2019 | Publicado en septiembre 2019

\section{RESUMEN}

El objetivo del estudio se centró en analizar la gestión de riesgos en las gerencias de seguridad industrial de la industria petrolera venezolana. Se tipificó como una investigación descriptiva, con diseño no experimental, transeccional y de campo. La población estuvo conformada por las cuatro (4) gerencias de seguridad industrial de la industria petrolera en occidente, cuyos sujetos informantes fueron gerentes $\mathrm{y}$ superintendentes. La técnica de recolección de datos fue la encuesta, y el instrumento un cuestionario con 69 reactivos y una escala de frecuencias de cinco (5) opciones de respuesta. Se utilizó el juicio de expertos para la validez del mismo y el coeficiente Alfa de Cronbach para su confiabilidad, cuyo valor de 0,863 demostró muy alta confiabilidad. Se aplicó como técnica para el análisis la estadística descriptiva, a través de la media aritmética $(\mathrm{X})$. Del análisis se comprobó buena gestión dirigida a la prevención de los riesgos.

Palabras clave: Prevención de riesgos, procesos de gestión, seguridad industrial

\section{ABSTRACT}

The objective of the study focused on analyzing risk management in the industrial security management of the Venezuelan oil industry. It was typified as a descriptive investigation, with non-experimental, transectional and field design. The population was made up of the four (4) industrial security management of the oil industry in the West of the country, whose reporting subjects were managers and superintendents. The data collection technique was the survey, and the instrument a questionnaire with 69 reagents and a frequency scale of five (5) response options. The expert judgment was used for its validity and Cronbach's alpha coefficient for its reliability, whose value of 0.863 demonstrated very high reliability. Descriptive statistics were applied as a technique for the analysis, through the arithmetic mean $\left(X^{-}\right)$. The analysis showed good management aimed at risk prevention.

Key words: Risk prevention, management processes, industrial safety 


\section{INTRODUCCIÓN}

Cuando se hace referencia a las empresas, cabe resaltar que estas abarcan diferentes espacios de producción implicando el campo industrial, el cual a su vez requiere de diversos servicios para llegar a ser operativos y cumplir con las responsabilidades asignadas, además, de las medidas de seguridad e higiene necesarias para cumplir con las responsabilidades correspondientes.

De este modo, al hablar de servicios, estos pueden ser de diversa índole según la naturaleza de la empresa, mencionándose, por ejemplo, los servicios de carácter energético como es el caso de la industria petrolera, ya que en este sector se presentan diversas causas y efectos en materia de riesgos; razones que justifican modelos de gestión apropiados para la operación de la misma.

Bajo este enfoque, es importante señalar y explicar las implicaciones teóricas del concepto de gestión entendido como un complejo integral que contempla un proceso de trabajo y organización, el cual permite la coordinación de esfuerzos para dar respuestas a los requerimientos de la empresa en cualquiera de sus aspectos estructurales (Villamayor, 2008). En este sentido, debe considerarse que la empresa puede estructurarse bajo diversas necesidades para optimizar su funcionamiento, uno de ellos, quizás uno de los más importantes en la actualidad se corresponde con evitar los riesgos.

Desde esta óptica, a nivel mundial, la gestión de riesgos representa un elemento de gran importancia en las diferentes organizaciones. Es considerada una herramienta para la mejora continua en la prevención de incidentes y accidentes de trabajo, así como de las enfermedades ocupacionales; cuyo objetivo es establecer estrategias para evitar que la salud de los trabajadores se deteriore con el paso del tiempo, incrementar el nivel de seguridad en las actividades además de la confianza por parte del trabajador hacia la empresa y viceversa.

En el mismo orden de ideas, la Comisión Venezolana de Normas Industriales (COVENIN) en su norma COVENIN 4001:2000 (2000) considera la gestión de riesgos como la aplicación sistemática de políticas, procedimientos y prácticas de gestión para analizar, valorar y evaluar los riesgos. De esta forma, se entiende entonces que la gestión de riesgos operacionales es un conjunto de actividades coordinadas para identificar, evaluar $\mathrm{y}$ controlar los riesgos a los cuales están expuestos los trabajadores en su medio ambiente laboral.

Debe señalarse que anteriormente, las organizaciones solo se limitaban a realizar acciones aisladas para dar cumplimiento con las diversas disposiciones legales y normativas en materia de seguridad y salud laboral, protegiendo al trabajador de posibles accidentes o enfermedades ocupacionales. En este sentido, su objetivo principal estaba dirigido al incremento de la producción y rentabilidad de la empresa.

En la actualidad, las organizaciones, especialmente las grandes empresas, han cambiado su visión, prestando mayor atención al recurso humano, evaluando los riesgos a los cuales puede estar expuesto el trabajador, fomentando su participación en la vigilancia del ambiente de trabajo, así como en el establecimiento de mejores prácticas de trabajo seguro, todo ello con la finalidad de mejorar la calidad de vida del trabajador. 
Es así como a nivel mundial, las empresas petroleras han convertido la gestión de riesgos en una actividad sistemática a fin de conservar los espacios en condiciones idóneas de operación satisfactoria, a través de la ejecución de trabajos basados en la sustitución, reparación, modificación, detección de defectos y prevención de los riesgos incipientes.

El propósito de lo referido anteriormente, según Grimaldi-Simons (2007), es lograr el cumplimiento de todos los servicios a tiempo, el cuidado adecuado de la empresa, en especial de los equipos que representan la parte operativa, como estrategia para evitar el riesgo, tendiente a prolongar la vida útil de los bienes, así como a obtener un rendimiento aceptable de los mismos durante más tiempo y reducir el número de fallas.

En tal sentido, para que los servicios ofrecidos por la industria petrolera sean exitosos, se deben cumplir ciertos objetivos, tales como evitar, reducir, corregir y en su caso, reparar las fallas que pueden ocasionar riesgos humanos o sobre los bienes. En efecto, disminuir la gravedad de las fallas permite evitar accidentes e incidentes, a fin de aumentar los niveles de productividad, aumentar la seguridad para las personas, conservar bienes productivos en condiciones seguras de operación, balanceando el costo, además de alcanzar o prolongar la vida útil de los bienes.

En este marco de referencia, de acuerdo a Grimaldi-Simonds (2007), debe considerarse que la ejecución de una gestión que permita evitar los riesgos en las empresas resulta cada día más compleja, debido a la tendencia que se corresponde con niveles de demandas progresivas, lo cual se traduce en aumentos significativos en la elevación operativa para cumplir con los requerimientos de una sociedad modernizada como la actual.

Estas situaciones, someten a la empresa a la necesidad de ser responsable con sus clientes a toda costa, a veces sin considerar de qué manera pueden evitar riesgos al aumentar su productividad. Por ello, las empresas a nivel mundial solicitan con mayor celeridad sistemas de gestión de riesgos, para de esa manera organizar cada una de las actividades a cumplir dentro de su producción.

Así, al hablar de las necesidades se debe establecer correspondencia con un sistema organizacional direccionado en cada uno de los pasos de producción, como: planificación, organización y ejecución; además, se requiere congruencia con respecto a las demandas modernas, de modo que se hace necesario un modelo de gestión propio de riesgos operacionales.

$\mathrm{Al}$ respecto, la norma OHSAS 18001:2007 (2008, p. 10) señala que "La organización debe establecer, implementar y mantener un(os) procedimiento(s) para la continua identificación de peligros, evaluación de riesgos y determinación de los controles necesarios".

Tomando en cuenta lo planteado, se hace mención a la industria petrolera venezolana, Petróleos de Venezuela S. A. (PDVSA), la cual constituye la principal fuente generadora de ingresos económicos al país; en la cual se han desarrollado las mejores prácticas de trabajo, elaboración de normas, procedimientos, análisis de riesgos, divulgación tanto de los riesgos a los cuales estará expuesto el trabajador como las lecciones aprendidas, tal y como lo establece la Ley Orgánica de Prevención Condiciones y Medio Ambiente de Trabajo (LOPCYMAT, 2005) en su artículo 56 
referido a deberes de los empleadores y empleadoras, numerales 3 y 4 en los cuales se establece lo siguiente:

Numeral 3. "Informar por escrito a los trabajadores y trabajadoras de los principios de la prevención de las condiciones inseguras o insalubres, tanto al ingresar al trabajo como al producirse un cambio en el proceso laboral o una modificación del puesto de trabajo e instruirlos y capacitarlos respecto a la promoción de la salud y la seguridad, la prevención de accidentes y enfermedades profesionales, así como también en lo que se refiere al uso de dispositivos personales de seguridad y protección".

Numeral 4. "Informar por escrito a los trabajadores y trabajadoras y al Comité de Seguridad y Salud Laboral de las condiciones inseguras a las que están expuestos los primeros, por la acción de agentes físicos, químicos, biológicos, meteorológicos 0 a condiciones disergonómicas o psicosociales que puedan causar daño a la salud, de acuerdo a lo establecido por el Instituto Nacional de Prevención, Salud y Seguridad Laborales (INPSASEL)."

Se puede decir entonces, que la gestión de riesgos se inicia desde el proceso de selección del personal, el cual al ser capacitado para ejecutar la actividad por la cual fue contratado, se le debe ayudar a desarrollar competencias para identificar, evaluar, controlar y divulgar los riesgos operacionales, así como las medidas de control; y finaliza cuando se realiza un seguimiento continuo sobre la prevención de los mismos.

En este sentido, las organizaciones deben realizar el mayor esfuerzo para la implementación de la gestión de riesgos a fin de disminuir la accidentalidad y enfermedades ocasionadas por el trabajo, así como crear ambientes armónicos y una cultura orientada hacia la prevención de los riesgos en todos los niveles jerárquicos de la empresa.

De lo anterior se destaca, que al implementar la gestión de riesgos otras áreas estarían beneficiadas, como lo son: disminución de los costos asociados a la ocurrencia de accidentes, incremento de la producción, reconocimiento de la empresa en el mercado y el nivel de calidad de vida del trabajador en su entorno laboral.

Ahora bien, según Espiñeira, Sheldon y Asociados (2008), en Venezuela han surgido grupos orientados a perjudicar mediante actos violentos los controles internos de la industria petrolera para cometer fraudes, sin dejar de mencionar que también han surgido fenómenos naturales que atentan contra los bienes y contra los mismos seres humanos. En función de ello, se comprende que es trascendental gestionar riesgos.

De acuerdo a estos autores, la gestión de riesgos es aplicable el caso mencionado anteriormente y su estudio se centra en la manera de proteger las organizaciones ante los eventos o situaciones, tanto del mundo como de la actualidad de cada país, protegiendo sus patrimonios y aportando un valor agregado.

En consonancia con lo reseñado, se observa que cada día, y con mayor frecuencia, diversas empresas están adoptando no solo medidas estratégicas dirigidas a enfrentar los riesgos, sino también técnicas o metodologías orientadas a los cambios, al parecer incumpliendo con normativas, leyes o regulaciones, ya que el objetivo final es dar resultados positivos a los problemas, cerrar brechas, inducir un mejoramiento, aumentar la productividad, 
buscar soluciones e inducir un control eficiente para lograr cambios significativos en los sistemas.

En este marco de referencia, Espiñeira et al. (2008) indican que a lo largo de los años la gestión de riesgos ha evolucionado, los gerentes han visto la necesidad de implantar nuevas tácticas, además de diferentes estrategias, para disipar los riesgos que crean problemáticas empresariales por causas financieras $u$ operacionales, y sobre todo por causas de la seguridad e integridad de los trabajadores. En general puede decirse, que la gestión de riesgos comprende la identificación, cuantificación, análisis y respuesta; estos cuatro pasos constituyen el análisis que se puede dar dentro de la organización.

En tal sentido, Cano, Rebollan y Lidon (2008) exponen que la gestión de riesgos corresponderá a acciones de planificación de respuesta y control de la evaluación del riesgo a lo largo del proyecto, la planificación de la respuesta al riesgo abarca: identificación de las áreas de riesgo potencial, análisis de riesgos principales y determinación de las respuestas ante riesgos.

Por su parte, Bravo y Sánchez (2006) agregan que puede definirse el riesgo como la incertidumbre que afecta negativamente el bienestar de la gente debido al hecho de representar la probabilidad de un cambio de resultado, en el cual intervienen varios aspectos en materia de seguridad, donde el proceso de identificación de peligros y evaluación de riesgos comprende varias etapas, utilizando diferentes herramientas para proveer orientación sobre las acciones a tomar para gestionar en forma efectiva, así como en el eficiente control de los riesgos para el personal, entorno $y$ empresa.
Al respecto, la duración como los plazos para completar todas las etapas de la gestión de riesgos dependen no solo del tamaño, sino también de la complejidad de la operación analizada, los cuales serán definidos y planificados por los asesores o encargados de la seguridad industrial, ambiente e higiene ocupacional, conjuntamente con los ingenieros y la participación de los supervisores de una actividad o proyecto a ejecutar.

Siendo así, resulta oportuna la necesidad de analizar la gestión de riesgos enfocada a las operaciones en las gerencias de seguridad industrial de la industria petrolera en occidente, dirigida a los diferentes tipos de riesgos inherentes a este tipo de actividad.

El análisis permitirá conocer en qué medida se relaciona la gestión del proceso con los resultados, tanto positivos como negativos, de las operaciones. La inquietud de realizar este análisis surge, porque de no darse una gestión efectiva de los riesgos enfocada en la seguridad de los trabajadores, traería consecuencias tanto a la empresa como a los empleados que llevan a cabo la actividad. De forma que no se efectuarían los procesos operacionales de manera eficiente y se pondría en riesgo la vida del personal involucrado, así como de la propia comunidad donde se llevan a cabo las actividades; además de evidenciarse el incumplimiento con procedimientos de trabajo.

Destacando que una gestión de riesgos deficiente, aumentaría considerablemente los índices de accidentalidad, serían inexistentes los parámetros mínimos de calidad de las operaciones y se propiciaría un incumplimiento con los clientes. Los tiempos de ejecución también se verían 
afectados al incurrir en riesgos durante las operaciones.

En relación a las gerencias de seguridad industrial de la industria petrolera en occidente, se plantea que el hecho de no darse una efectiva gestión de todos los riesgos involucrados en la ejecución de las operaciones, implicaría el estar aplicando prácticas inadecuadas, llevando a cometer actos inseguros, estando bajo condiciones inseguras de trabajo, con utilización de herramientas y equipos en condiciones inadecuadas, trabajar sin parámetros mínimos de seguridad ni medidas preventivas en la utilización de los productos y equipos a utilizar.

Las principales causas que llevan a una deficiente gestión de los riesgos dentro de las organizaciones, en este caso las empresas petroleras, son los constantes cambios debido a la globalización, donde actualmente se da mayor valor a la productividad y a la continuidad de las operaciones, dejando en otro plano el elemento de seguridad industrial, ambiente e higiene ocupacional, que a juicio del investigador es piedra angular para la prolongación de cualquier actividad en la industria petrolera.

Así, se puede decir que una empresa cuando no controla de forma efectiva y eficiente los riesgos asociados a sus operaciones, a los cuales están expuestos sus trabajadores resulta incierto en qué momento puede producirse una ausencia laboral, lo que comprometería en algún grado la continuidad de las actividades por falta de personal. Asimismo, puede argumentarse que el hecho de controlar las operaciones bajo los estándares de seguridad industrial exitosos, permite obtener más resultados positivos a cualquier nivel de la organización, ya que se posee personal capacitado, se cuenta con maquinaria adecuada y recursos necesarios para lograr minimizar el impacto producido por la exposición de los trabajadores a los agentes de peligro.

A largo plazo, las consecuencias o efectos negativos para una organización se verían reflejados en aspectos económicos por los gastos que conllevan un accidente $o$ lesión ocupacional, pérdidas de tiempo durante las operaciones, ausencias laborales y hasta pérdidas de vidas humanas, solo por mencionar algunas.

En concordancia con estas reflexiones, se acota que otro aspecto incidente en el interés por analizar la gestión de riesgos en la industria petrolera venezolana, corresponde a la cantidad de accidentes reportados solo en la actividad económica referida a la extracción de petróleo crudo y gas natural donde hubo un incremento del $47 \%$ del año 2017 al 2018, según datos publicados por el INPSASEL (2019). Es preciso mencionar que, al ocurrir un accidente, dependiendo de la magnitud del mismo, es afectado además del trabajador, el entorno familiar, la organización, los compañeros de trabajo, la comunidad y el medio ambiente.

Por ello, es importante conocer hasta dónde se están cumpliendo las leyes, normas y procedimientos, tanto nacionales como internacionales, que rigen la seguridad y salud laboral, específicamente en cuanto a la gestión de riesgos, considerando particularmente a Venezuela, donde la industria petrolera se enfrentaría a una multa por una cantidad de dinero considerable debido al incumplimiento de las mismas, así como las consecuencias sobre su imagen a nivel internacional. Igualmente, es preciso conocer el compromiso de los líderes de las organizaciones que dirigen la empresa. 
En el caso de las gerencias de seguridad industrial de la industria petrolera en occidente, es de interés conocer cuál es su situación actual en la gestión de riesgos con respecto a la identificación de los factores de riesgo operacional, entre ellos los riesgos físicos, químicos, biológicos, ergonómicos, psicosociales y ambientales.

Asimismo, se considera importante abordar la descripción de los elementos clave de la gestión de riesgos, tales como la capacitación del personal, los procesos de registro y documentación, equipos del lugar de trabajo y forma en que llevan a cabo la prevención de los riesgos; adicional a esto, examinar cómo llevan a cabo el proceso de gestión de riesgos operacionales considerando aspectos como identificación del riesgo, evaluación del riesgo, planes de respuesta, manejo de las contingencias y control del riesgo operacional.

Los resultados de la investigación, permitieron visualizar una manera factible de corregir desviaciones, además de crear acciones estratégicas efectivas que les permitan mitigar los riesgos inherentes a la actividad; así mismo, satisfacer no solo las necesidades de los clientes, sino también prestar un mejor servicio, brindando un ambiente satisfactorio de trabajo para empleados, empresa y comunidad. Se destaca, que en las últimas décadas se ha considerado el factor humano como activo indispensable dentro de las grandes corporaciones, allí radica la importancia de brindar un factor de seguridad óptimo para mantener la continuidad de las operaciones que el hombre gestiona.

\section{Gestión del riesgo}

Bravo y Sánchez (2006) definen el riesgo como la incertidumbre que afecta de forma negativa el bienestar de la gente, debido a que representa la probabilidad de un cambio de resultado. En el mismo orden de ideas, Amendola (2006) explica que el riesgo es una medida de la extensión en la cual un resultado que se espera, puede desviarse o desvirtuarse, tomando en cuenta diferentes variables. Para Cano et al. (2008) el riesgo es todo aquello que caracteriza o da forma a las situaciones, donde el resultado final de una actividad es probable que cambie o de un resultado inesperado.

Bajo esta perspectiva, para Cortés (2007), el riesgo representa aquello que puede ocasionar un daño o deteriorar la calidad de vida de cada individuo o del colectivo, fundamentado en factores externos y propios de las actividades que se ejecutan, los mismos pueden ser controlados si se identifica el origen y las consecuencias de los mismos. Una vez lograda la identificación, se hace énfasis en que existen agentes de riesgos que pueden ser tolerados por el trabajador y su entorno.

Por su parte, Hernández, Malfavon y Fernández (2012) definen el riesgo como la posibilidad de pérdida, así mismo, es el grado de probabilidad que se puede tener de las pérdidas. Para los autores existen riesgos puros, los cuales llevan solo a obtener pérdidas, y riesgos especulativos son los que llevan a una organización a obtener ganancias o pérdidas. En el mismo orden de ideas, indican que para llevar a cabo un eficiente control de los riesgos es necesario tomar en cuenta las siguientes consideraciones: detectar el peligro, eliminar el peligro y por ende el riesgo, reducir el riesgo desde el $100 \%$ hasta un valor de $0 \%$ o casi igual, lograr la transferencia del riesgo, es decir, asegurarlo cuando se llegue a una probabilidad de $0 \%$. 
Ahora bien, Espiñeira et al. (2008) asocian este término con la gestión, planteando que la gestión de riesgos ha evolucionado, los gerentes han visto la necesidad de implantar nuevas tácticas y estrategias para disipar los riesgos que crean problemáticas en las empresas por causas operacionales. Con respecto a este concepto, Heerkens (2007) opina desde una visión general, que la gestión de riesgos comprende las etapas: identificación, cuantificación, análisis y respuesta. Estos cuatro pasos comprenden el análisis que se puede dar dentro de la organización.

En este orden de ideas, las Normas Industriales COVENIN 2270:2002 (2002), establecen que el proceso de gestión de riesgos es la aplicación sistemática de políticas, procedimientos y prácticas de gestión para analizar, valorar, así como evaluar los riesgos organizacionales en el área de operaciones.

Por su parte, López (2013) plantea que la gestión de riesgos es un proceso integral que comprende determinadas funciones ejecutadas por los niveles de gestión, para asignar recursos y coordinar el trabajo dentro de la organización, con la finalidad de preservar la salud física y mental de los trabajadores, así como la población, bienes y ambiente.

A criterio del investigador, la gestión de riesgos se constituye en la aplicación sistemática de políticas, procedimientos y prácticas de gestión para analizar, valorar, así como evaluar los riesgos en las operaciones de la industria petrolera en occidente.

\section{MÉTODO}

Se trató de un estudio de carácter descriptivo, con un diseño no experimental, transeccional y de campo. La población quedó conformada por las cuatro (4) gerencias de seguridad industrial de la industria petrolera en occidente, las cuales son: Costa Occidental, Lago, Costa Oriental y Sur Lago Trujillo, cuyos sujetos informantes fueron los gerentes y superintendentes, conformando una población de 14 unidades, a quienes se le aplicó una encuesta a través de un cuestionario conformado por 69 ítems con escala de frecuencia. El instrumento diseñado se sometió a la evaluación de dos (2) expertos metodológicos para avalar la construcción, y tres (3) expertos en el área de gerencia de seguridad para la validación del contenido. Luego se determinó su confiabilidad por medio del coeficiente Alfa de Cronbach, cuyo valor resultante fue de 0,863 indicando que el mismo posee muy alta confiabilidad, siendo apto para su aplicación.

Para procesar los resultados de la aplicación del cuestionario, se recurrió al método de la estadística descriptiva, específicamente se empleó la medida de tendencia central denominada media aritmética $(\overline{\mathrm{X}})$ o promedio. Para tal efecto, el investigador diseñó un baremo, tal como se muestra en el cuadro 1. 
Cuadro 1. Baremo para la interpretación de la media aritmética

\begin{tabular}{lll}
\hline INTERVALO & CATEGORÍA & DESCRIPCIÓN \\
\hline $4.21-5$ & Muy alta presencia & $\begin{array}{l}\text { Indica muy buena gestión de } \\
\text { riesgos operacionales/Fortaleza }\end{array}$ \\
$3.41-4.20$ & Alta presencia & $\begin{array}{l}\text { Indica buena gestión de riesgos } \\
\text { operacionales/Fortaleza }\end{array}$ \\
$2.61-3.40$ & Moderada presencia & $\begin{array}{l}\text { Indica regular gestión de riesgos } \\
\text { operacionales/Debilidad }\end{array}$ \\
$1.81-2.60$ & Baja presencia & $\begin{array}{l}\text { Indica mala gestión de riesgos } \\
\text { operacionales/Debilidad }\end{array}$ \\
\hline
\end{tabular}

\section{RESULTADOS Y DISCUSIÓN}

Como se evidencia en la tabla 1 , la media obtenida $(4,04)$ para la dimensión prevención de los factores de riesgo en la gestión de riesgos, implica alta presencia en las actividades ejecutadas con este fin en las gerencias de seguridad industrial de la industria petrolera en occidente, delineándola como una fortaleza para la gestión realizada por estas gerencias.
Este resultado, de alta presencia de la dimensión, contrasta con lo expuesto por Hernández et al. (2012), para quienes el riesgo representa la posibilidad de pérdida y el grado de probabilidad de estas pérdidas, la exposición a una posibilidad de accidente, es definido como correr el riesgo y depende directamente de un acto o condición insegura.

Tabla 1. Dimensión: prevención de los factores de riesgos operacionales

\begin{tabular}{ccc}
\hline SUB-DIMENSIONES & MEDIA & CATEGORÍA \\
\hline FACTORES DE RIESGO FÍSICO & 4,16 & Alta presencia/fortaleza \\
FACTORES DE RIESGO QUÍMICO & 4,27 & Muy alta presencia/fortaleza \\
FACTORES DE RIESGO BIOLÓGICO & 3,86 & Alta presencia/fortaleza \\
FACTORES DE RIESGO PSICOSOCIALES & 3,90 & Alta presencia/fortaleza \\
FACTORES DE RIESGO ERGONÓMICO & 4,00 & Alta presencia/fortaleza \\
DIMENSIÓN & $\mathbf{4 , 0 4}$ & Alta presencia/fortaleza \\
\hline
\end{tabular}

A tal efecto, se demuestra como en las gerencias analizadas se están gestionando las condiciones de trabajo de manera adecuada, previniendo en alta medida los factores que generan riesgos operacionales, reconociendo la necesidad de que sus instalaciones, equipos y materiales de trabajo estén en condiciones adecuadas para preservar la salud de los empleados, con el fin de ofrecer un servicio exitoso y cumplir con los objetivos propuestos. 
En este recorrido del análisis de resultados, se presentan a continuación los obtenidos para cada uno de los indicadores que conforman la segunda dimensión denominada: Elementos clave de la gestión de riesgos. De esta manera, en la tabla 2, se presenta el resumen para esta dimensión, reflejando una media para la dimensión de 4,46. Esto indica en las gerencias bajo estudio, que se da alta presencia a los elementos clave de la gestión de riesgos, siendo esto una fortaleza en cuanto a los aspectos medidos para la gestión analizada.

Tabla 2. Dimensión: elementos clave de la gestión de riesgos operacionales

\begin{tabular}{ccc}
\hline INDICADORES & MEDIA & CATEGORÍA \\
\hline CAPACITACIÓN DEL PERSONAL & 4,38 & Muy alta presencia/fortaleza \\
REGISTRO Y DOCUMENTACIÓN & 4,60 & Muy alta presencia/fortaleza \\
EQUIPOS DEL LUGAR DE TRABAJO & 4,07 & Alta presencia/fortaleza \\
PREVENCIÓN DE LOS RIESGOS & 4,79 & Muy alta presencia/fortaleza \\
DIMENSIÓN & $\mathbf{4 , 4 6}$ & Muy alta presencia/fortaleza
\end{tabular}

Los resultados reflejan una alta presencia en cuanto a los elementos clave de la gestión de riesgos operacionales, lo cual valida lo planteado por Cortés (2007), para quien las actuaciones más eficaces para combatir los riesgos deben partir del conocimiento de éstos. Tomando en cuenta estos resultados, a criterio del investigador, puede describirse la alta presencia de los elementos clave de la gestión de riesgos en las gerencias analizadas, lo que permite prever las necesidades y aspectos tanto perfectibles como conservables.
Adicionalmente, se muestran los resultados de la tercera dimensión, denominada proceso de gestión de riesgos. A tal efecto, en la tabla 3 se resumen los resultados obtenidos para la dimensión referida, evidenciando una media de 4,55. Este valor implica muy alta presencia del proceso de gestión de riesgos en las gerencias reseñadas, delineándola como fortaleza de la gestión que desarrollan dichas gerencias.

Tabla 3. Dimensión: proceso de gestión de riesgos

\begin{tabular}{ccc}
\hline INDICADORES & MEDIA & CATEGORÍA \\
\hline IDENTIFICACIÓN DEL RIESGO & 4,70 & Muy alta presencia/fortaleza \\
EVALUACIÓN DEL RIESGO & 4,44 & Muy alta presencia/fortaleza \\
PLANES DE RESPUESTA & 4,34 & Muy alta presencia/fortaleza \\
MANEJO DE LAS CONTINGENCIAS & 4,60 & Muy alta presencia/fortaleza \\
CONTROL DEL RIESGO OPERACIONAL & 4,69 & Muy alta presencia/fortaleza \\
DIMENSIÓN & $\mathbf{4 , 5 5}$ & Muy alta presencia/fortaleza \\
\hline
\end{tabular}


Estos resultados, validan las Normas Industriales COVENIN 2270:2002 (2002), en las cuales se define el proceso de gestión de riesgos como la aplicación sistemática de políticas, procedimientos y prácticas de gestión para analizar, valorar, así como evaluar los riesgos organizacionales en el área de operaciones

De acuerdo a lo evidenciado, en las gerencias de seguridad industrial de la industria petrolera en occidente se concede muy alto reconocimiento al proceso de gestión de riesgos, asumiéndolo como un conjunto de actividades coordinadas para identificar, evaluar y controlar los riesgos a los cuales están expuestas todas las operaciones en su medio de desempeño.

Para finalizar el análisis de los resultados, en la tabla 4 se muestran los valores obtenidos para la variable gestión de riesgos operacionales. Al detalle, se visualizó alta presencia de los factores de riesgo, y muy alta presencia en los elementos clave y proceso de gestión de riesgos, teniendo fortalezas en todas sus dimensiones.

Tabla 4. Variable: gestión de riesgos operacionales

\begin{tabular}{ccc}
\hline DIMENSIÓN & MEDIA & CATEGORÍA \\
\hline $\begin{array}{c}\text { Prevención de los factores de riesgo } \\
\text { operacionales }\end{array}$ & 4,00 & Alta presencia/fortaleza \\
$\begin{array}{c}\text { Elementos claves de la gestión de riesgos } \\
\text { operacionales }\end{array}$ & 4,46 & Muy alta presencia/fortaleza \\
$\begin{array}{c}\text { Proceso de gestión de riesgos operacionales } \\
\text { VARIABLE }\end{array}$ & 4,55 & Muy alta presencia/fortaleza \\
& $\mathbf{4 , 3 4}$ & $\begin{array}{c}\text { Muy alta presencia/fortaleza MUY } \\
\text { BUENA GESTIÓN }\end{array}$ \\
\hline
\end{tabular}

Esta calificación, de muy buena gestión, posee muy alta congruencia con lo dicho en la definición conceptual de la variable dada por López (2013), para quien la gestión de riesgos es un proceso integral que comprende determinadas funciones ejecutadas por los niveles de gestión, para asignar recursos y coordinar el trabajo dentro de la organización, con la finalidad de preservar la salud física y mental de los trabajadores, así como la población, bienes y ambiente.

En virtud de estos hallazgos, donde se cataloga la gestión como muy buena, se considera que hay muy alta coincidencia con la posición del investigador, para quien la gestión de riesgos se constituye en la aplicación sistemática de políticas, procedimientos y prácticas de gestión para analizar, valorar, así como evaluar los riesgos en las operaciones de la industria petrolera en occidente.

\section{CONCLUSIONES}

En lo concerniente al primer objetivo, en el cual se diagnosticó la prevención de los factores de riesgo presentes en la gestión, se conoció como estas gerencias poseen alta presencia en la prevención de los factores considerados en esta investigación. En específico, se detectó alta presencia en la prevención de los factores de riesgo físicos, biológicos, psicosociales y ergonómicos, resaltando la referida a los factores de riesgo químico, categorizados con muy alta presencia, indicando fortalezas de la gestión en cuanto a su prevención. 
Sin embargo, a juicio del investigador, aun cuando se arriba a la categoría de alta presencia en la prevención de los factores de riesgo, se detectó la existencia de ciertos aspectos que pueden ser reforzados, motivo por el cual resulta prioritario actuar contra los riesgos que provocan accidentes de trabajo o enfermedades, debido a que estos pueden afectar las diferentes actividades que se realizan en las empresas petroleras, disminuyendo el rendimiento y la calidad de sus servicios.

Para el segundo objetivo, donde se describieron los elementos clave de la gestión de riesgos, se evidenció que estos elementos reflejaron muy alta presencia. No obstante, de manera similar al objetivo anterior, se evidenciaron oportunidades de mejora, por lo que las gerencias analizadas deben tener como base el desempeño de actividades destinadas a contribuir de manera favorable con el desarrollo de las operaciones bajo una consideración efectiva de su gestión.

En cuanto al tercer objetivo, orientado a examinar el proceso de gestión de riesgos, se pudo evidenciar como este proceso arriba a la categoría de muy alta presencia, indicando que la gestión posee fortalezas en estos aspectos. Es importante acotar que, de manera análoga a los objetivos anteriores, se conoció la existencia de oportunidades de mejora, por lo cual es importante que las gerencias analizadas identifiquen las pérdidas potenciales, a fin de asegurar que se consideren las acciones requeridas para aumentar las posibilidades de éxito, buscando reducir o eliminar los riesgos.

Con respecto al objetivo general, cuya orientación se centró en analizar la gestión de riesgos, se evidenció muy alta presencia de esta gestión, indicando que estas gerencias de seguridad industrial de la industria petrolera en occidente, utilizan un conjunto de procedimientos sistemáticos que permiten identificar, medir y evaluar riesgos mediante la aplicación de normas tangibles para el control de pérdidas.

\section{REFERENCIAS}

Bravo, 0. y Sánchez, M. (2006). Gestión integral de riesgos. Colombia: Editorial B \& S

Cano, J., Rebollan, R. y Lidon, L. (2008). Apuntes de gestión de proyectos. Tercera Edición. Valencia: Editorial Cisne

Cortés, J. (2007). Seguridad e higiene del trabajo. Técnicas de prevención de riesgos laborales. Madrid: Editorial TEBAR

Espiñeira, Sheldon y Asociados (2008). Boletín de asesoría gerencial. ¿Cómo analizar la madurez en la administración del riesgo operacional? № 13. Venezuela. Recuperado de https://docplayer.es/14710437-

Boletin-de-asesoria-gerencial-comoalcanzar-la-madurez-en-laadministracion-del-riesgooperacional.html

Grimaldi-Simonds, R. (2007). La seguridad industrial. México: Editorial Alfaomega

Heerkens, G. (2007). Gestión de proyectos. España: Editorial McGrawHill

Hernández, A., Malfavon, N. y Fernández, G. (2012). Seguridad e higiene industrial. México: Editorial Limusa

LOPCYMAT (2005). Ley Orgánica de Prevención, Condiciones y medio ambiente de trabajo. Instituto Nacional de prevención, salud y seguridad Laborales. Recuperado de http://www.inpsasel.gob.ve/moo_ne ws/lopcymat.html 
López, I. (2013). Prevención de riesgos laborales. Primera edición. España: Editorial Universidad Internacional de la Rioja S.A.

Norma COVENIN 2270:2002. (2002). Comité de Higiene y Seguridad en el Trabajo. Guía para su funcionamiento. Tercera revisión. Caracas: FONDONORMA

Norma COVENIN 4001:2000. (2000). Sistema de gestión de seguridad $e$ higiene ocupacional (SGSHO). Requisitos. Caracas: FONDONORMA

Norma OHSAS 18001:2007. (2008). Sistemas de gestión de seguridad y salud ocupacional. Requisitos. Venezuela: FONDONORMA

Villamayor, C. (2008). Saneamiento ambiental. México: Editorial de la Universidad Autónoma de Chiapas 